\title{
Evidence Linking Mental Health with Obesity and Metabolic Syndrome: The Role of Inflammation
}

\author{
Alanna A. Morris • Viola Vaccarino \\ Published online: 27 September 2013 \\ (C) Springer Science+Business Media New York 2013
}

published that define the criteria for the diagnosis of MetS; however, the most widely used come from the 2001 Third Report of the National Cholesterol Education Program Adult Treatment Panel (NCEP-ATP III), because it employs easily measurable risk factors [3]. The guidelines have been revised since the original report for stricter criteria [4] and currently include having three or more of the following five cardiovascular disease (CVD) risk factors: 1) central obesity (waist circumference: men $\geq 102 \mathrm{~cm}$; women $\geq 88 \mathrm{~cm}$ ); 2) elevated triglycerides ( $\geq 150 \mathrm{mg} / \mathrm{dl}$ ); 3 ) diminished high-density lipoprotein (HDL) cholesterol (men $<40 \mathrm{mg} / \mathrm{dl}$; women $<50 \mathrm{mg} /$ dl); 4) systemic hypertension ( $\geq 130 / \geq 85 \mathrm{~mm} \mathrm{Hg}$ ); and 5) elevated fasting glucose $(\geq 100 \mathrm{mg} / \mathrm{dl})$. MetS is widely recognized for its association with increased CVD morbidity and mortality $[5 \bullet \cdot, 6]$; however, its association with disorders of mental health is being increasingly documented. Numerous reports have confirmed that persons with mental illness have an increased prevalence of cardiometabolic risk factors that adversely impact CVD morbidity and mortality, and in some cases, treatment with psychotropic medications can actually increase that risk.

Central or abdominal obesity is considered to be the most prevalent manifestation of MetS and is of central importance in the clinical diagnosis, because it is a marker of "dysfunctional adipose tissue." [7] Adipose tissue is now recognized as an endocrine organ that secretes numerous immunomodulatory factors. Abdominal adiposity can be further characterized by excess visceral (VAT) and subcutaneous (SAT) adipose tissues, which likely differ in their metabolic activity and functional consequences, as VAT seems to be a significant source of inflammatory signals related to macrophage infiltration into adipose tissue $[8,9]$. For any given amount of total body fat, individuals with a selective excess of VAT are at substantially higher risk of being characterized by insulin resistance and the features of the MetS [7, 10]. Recent data suggest that in obese individuals, VAT is more closely
A. A. Morris $(\bowtie)$

Division of Cardiology, Emory University School of Medicine, 1364 Clifton Road Suite D403B, Atlanta, GA 30322, USA

e-mail: aamorr3@emory.edu

V. Vaccarino

Department of Epidemiology, Emory University School of Public Health, Atlanta, GA, USA 
associated with an adverse metabolic, dyslipidemic, and atherogenic profile, whereas SAT demonstrates a more benign phenotype characterized by modest associations with inflammatory biomarkers, but no association with dyslipidemia, insulin resistance, or atherosclerosis [11•].

A chronic low-grade inflammatory state is associated with the presence of the MetS, reflecting increased production of the acute phase reactant $\mathrm{C}$-reactive protein (CRP), and proinflammatory cytokines including interleukin-6 (IL-6) and tumor necrosis factor-alpha (TNF- $\alpha$ ) among others. CRP is the most well-recognized biomarker of systemic inflammation, because it enhances traditional prognostic information for CVD risk and outcomes in patients with MetS [12, 13]. The contribution of IL- 6 and TNF- $\alpha$ to atherogenic processes is less well understood than the role of CRP. However, multiple studies have shown that IL- 6 and TNF- $\alpha$ are associated with incident CVD and mortality [14, 15] and enhance risk prediction for total CVD mortality in addition to traditional risk factors and CRP [16 $]$.

Levels of CRP, IL-6, and TNF- $\alpha$ are increased in persons with MetS $[13,17,18]$ and may contribute to the excess CVD risk in this population. The low-grade inflammatory state that characterizes MetS is attributed to the increased activity of macrophages in the adipose tissue of obese individuals [19]. Macrophages increase in number in the adipose tissue of obese individuals, and these macrophages are responsible for most of the cytokine production in obese adipose tissue. In fact, these macrophages account for most of the obesityassociated TNF- $\alpha$ and significant amounts of IL- 6 secreted from the adipose tissue of obese individuals. The increased production of TNF- $\alpha$ and IL- 6 in obese adipose tissue, in addition to other proinflammatory cytokines, further induces CRP secretion from the liver and stimulates the hypothalamic-pituitary-adrenal (HPA) axis, leading to a vicious cycle of worsened central obesity, hypertension, and insulin resistance [20].

Adipose tissue also secretes a number of bioactive peptides termed "adipokines," which play a central role in energy and vascular homeostasis, as well as immunity. With increasing adiposity, the proinflammatory adipokine leptin is overproduced promoting insulin resistance and other metabolic abnormalities, while the anti-inflammatory and insulinsensitizing adipokine adiponectin is decreased [21]. Leptin levels are higher in obese individuals, partly due to overproduction, and partly due to leptin resistance in a similar way that patients with type 2 diabetes are resistant to insulin $[22 \bullet \bullet, 23]$. Leptin has direct proinflammatory effects and is known to activate circulating monocytes in addition to upregulating in vivo production of IL-6 and TNF- $\alpha$ [24]. Adiponectin exerts beneficial cardioprotective effects at every stage of the atherosclerotic process, including suppression of vascular-smooth muscle cell proliferation and migration and inhibition of macrophage-to-foam cell transformation [25].
Adiponectin also has potent anti-inflammatory and antioxidant properties, including inhibition of TNF- $\alpha$-induced superoxide radical generation in endothelial cells [26].

Systemic inflammation is increasingly implicated in the pathogenesis of neuropsychiatric disorders. Inflammatory molecules and cells are fundamental to the maintenance of central nervous system (CNS) and neuronal integrity but also can be destructive when the inflammatory response is overactive or sustained $[27 \cdot \bullet$. Peripheral inflammatory cytokines, such as those circulating as part of the MetS, may induce loss of integrity of the blood-brain barrier (BBB) and gain access to the CNS via leaky regions around endothelial cell tight junctions, allowing transport of peripheral inflammatory signals into the brain [28]. Obesity and MetS are both highly prevalent in patients with neuropsychiatric disorders, such as depression, anxiety, and schizophrenia. However, it is not clear whether this association is due to behaviors in patients with mental illness that promote weight gain, or whether the inflammation related to obesity further promotes the symptoms of mental illness. However, increasing data confirm that chronic immune activation contributes to the immense burden of neuropsychiatric disorders, and obesity-related inflammation may provide a link between MetS and mental illness. In this review, we examine the pertinent literature that explores the inflammatory link between mental health and obesity in the MetS.

\section{Depression}

Depression is one of the most common psychiatric illnesses in the world. It is estimated that 1 in 5 U.S. adults have at least one episode of major depression in their lifetime, and that depression is the leading cause of disability in the U.S. for adults aged 15 to 44 years [29, 30]. Depression and obesity share overlapping psychosocial and pathophysiological etiologies, and an association between the two conditions has been seen in many population-based studies [22••, $31 \bullet, 32 \bullet \cdot]$. Studies have also shown a higher prevalence of depression in patients with MetS [33-35, 36•], and it has been postulated that obesity-related inflammation may be the underlying link. For instance, depressive symptoms and MetS were analyzed in 652 women in the Women's Ischemia Syndrome Evaluation (WISE) study [37]. After adjusting for demographic factors, lifestyle and functional status, depressive symptoms were associated with $\sim 60 \%$ increased odds for MetS compared with no depression. Furthermore, women with depressive symptoms had 2.6 times higher risk of CVD over a median follow-up of 5.9 years. However, adding MetS to the multivariable analysis only decreased the risk of CVD associated with depression by $7 \%$, showing that MetS does not fully explain the association between depression and risk of CVD. Other epidemiologic cohort studies have investigated whether 
there is a bidirectional association between depression and MetS. A recent meta-analysis that included 9 studies $(\mathrm{N}=$ 26,936 with 2,316 new-onset depression case subjects) reported an adjusted OR 1.49 [1.19-1.87] with depression as the outcome, and 4 studies $(\mathrm{N}=3,834$ with 350 MetS case subjects) reported an adjusted OR 1.52 [1.2-1.91] with MetS as the outcome [38]. These results support the concept of a bidirectional association between depression and MetS.

Major depression involves complex interactions between genes and the environment that converge in the brain $[39,40]$. Numerous mechanisms have been implicated, including dysfunction of monoaminergic systems [41] and dysregulation of the HPA axis $[42,43]$. Inflammatory biomarkers and cytokines have been shown to influence many of these pathways [44]. Of the biomarkers that have been examined in depressed individuals, CRP and IL-6 are the most extensively studied. Numerous analyses have reported higher levels of CRP [31•, 44, 45., 46-49] in individuals with depressive symptoms. Furthermore, several reports have shown that the association between CRP and depression differs based on gender and race/ethnicity $[47,48,50,51 \bullet, 52 \cdot]$, as well as the subtype of depressive symptoms $[45 \cdot, 53]$. For instance, our analysis of 512 participants in the Morehouse and Emory Team up to Eliminate Health Disparities (META-Health) Study showed white women with mild to severe depressive symptoms had higher levels of CRP compared with those with minimal to no depressive symptoms [52•]. However, there were no differences in levels of CRP by severity of depressive symptoms in white men or African Americans of either sex. Studies also have confirmed an association between higher levels of IL-6 and depressive symptoms [49, 54-57]. Similar to CRP, a recent meta-analysis confirmed that the association of IL-6 and depression is influenced by gender and obesity [58]. The meta-analysis found that very few studies provided data partitioned by gender, but the relationship between IL-6 and depression was significant for those samples comprised only of women $(n=13)$, whereas there was no association in those samples comprised only of men. There may be a genetic component to the association of IL-6 levels and depressive symptoms. Analyses of 188 male twins from the Vietnam Era Twin Registry found a significant correlation between IL-6 and depressive symptoms, and after adjustment for other risk factors, genetic factors accounted for approximately $93 \%$ of the covariation between depressive symptoms and IL-6 [54].

The association of TNF- $\alpha$ with depressive symptoms has not been as clearly defined as with other biomarkers. A few analyses have noted higher levels of TNF- $\alpha$ in depressed subjects [36•,59•], whereas others have shown no association $[33,60]$. A recent meta-analysis of 13 studies confirmed significantly higher concentrations of TNF- $\alpha$ in depressed subjects compared with controls [61•]. Similar to findings with CRP, reports have shown that the association between TNF- $\alpha$ and depression may differ based on the subtype of depressive symptoms [36•]. Furthermore, recent trials using the TNF- $\alpha$ antagonist infliximab to reduce depressive symptoms in patients with treatment-resistant depression determined that baseline concentrations of TNF- $\alpha$ and its soluble receptors were significantly higher in infliximabtreated responders compared to nonresponders [62・•]. To date, relatively few studies have examined levels of TNF- $\alpha$ in depressed subjects with MetS. Capuron et al. did not note a correlation of TNF- $\alpha$ with depressive symptoms in 323 male twins with and without MetS [33]. Similarly, Zeugmann et al. examined 70 inpatients with major depressive disorder and did not find a relationship between MetS and TNF- $\alpha$ levels [63].

Fewer studies have examined the relationship between depression and the adipokines. Both low [64, 65] and high $[66,67]$ levels of leptin have been associated with depressive symptoms. Studies from our group found the association of depression and leptin is mediated by adiposity, with body mass index (BMI) accounting for $61 \%$ of the variance in leptin levels $[22 \bullet \cdot]$. Compared with those with minimal depression, participants with moderate to severe depressive symptoms and low to normal weight had lower levels of leptin, whereas participants with moderate to severe depressive symptoms who were overweight and obese had higher levels of leptin. Our findings suggest that leptin insufficiency may occur in a subset of depressed patients, whereas the high leptin levels observed with obesity may be indicative of leptin resistance in other patients. Also similar to CRP and IL-6, the association between leptin and depression may differ based on the subtype of depressive symptoms. A recent analysis by Chirinos et al. found that leptin was significantly associated with somatic depressive symptoms, but not total or cognitive symptoms in 135 participants with the MetS [68•]. Relatively small studies have shown lower levels of adiponectin in depressed individuals [69, 70, 71•], and an analysis from Zeugmann et al. also confirmed lower levels of adiponectin in depressed patients with MetS compared with depressed patients without MetS [63]. A study of 2,359 diabetic youth noted higher levels of adiponectin, leptin, CRP in type 1 diabetic participants with greater depressive symptoms [72•]. However, none of the markers were significantly associated with depression after adjustment for demographic and clinical covariates.

\section{Schizophrenia}

Multiple large studies have estimated a prevalence of MetS in patients with schizophrenia that is two to three times higher than the general population $[73,74]$. This clustering of CVD risk factors in schizophrenic patients has been attributed to unhealthy lifestyle, including poor diet, smoking, and sedentary behavior. However it also is well recognized that 
antipsychotic drugs cause weight gain as an adverse side effect, further increasing the risk of obesity related illnesses, such as diabetes and unfavorable lipid profile. In fact, a recent study of MetS in patients with schizophrenia showed that the incidence of new cases of MetS after three years in those started on second-generation antipsychotics was over twice the incidence of those treated with first-generation antipsychotic agents $(27.8 \%$ vs. $13.1 \%)$. Moreover, in patients without MetS at baseline, the risk of developing MetS was 3.6-fold greater in patients started on second-generation antipsychotics [75]. Because of the high prevalence of CVD risk factors, and an even higher incidence of developing MetS after starting antipsychotic medications, consensus guidelines recommend appropriate baseline metabolic screening for patients treated with second-generation antipsychotics and ongoing monitoring of parameters, including blood pressure, height and weight to calculate BMI, waist circumference, and fasting plasma lipids and glucose [76].

There has been considerable interest in the contribution of proinflammatory cytokines to the neuropathogenesis of schizophrenia. Uncontrolled activity of proinflammatory cytokines, biomarkers, and microglia, resident macrophages that act as the first and main immune defense in the CNS, can induce schizophrenia in patients with underlying genetic vulnerability [77]. Elevated levels of CRP have been observed in multiple observational and longitudinal studies, and a recent meta-analysis confirms a prevalence of elevated CRP in $28 \%$ of patients with schizophrenia and related disorders [78]. Furthermore, the relationship between CRP and schizophrenia has remained significant even after controlling for important confounding CVD risk factors, such as age, BMI, and smoking status [79]. Multiple small studies have shown higher levels of IL- 6 in the serum as well as the CSF in schizophrenic patients compared with healthy controls [80, 81]. In addition, specific investigations have revealed a relationship between various IL-6 gene polymorphisms and higher IL-6 blood levels in patients with schizophrenia [80, 82]. A recent meta-analysis confirmed that levels of TNF- $\alpha$ are elevated in acute exacerbations of schizophrenia and remain elevated following antipsychotic treatment [83].

There has been considerable interest in the association of schizophrenia with the adipokines given the high incidence of adverse effects that antipsychotic medications have on food intake, weight gain, and other metabolic parameters. Analyses have shown higher levels of leptin in schizophrenic patients with MetS compared with healthy controls [84], and polymorphisms in the leptin gene are thought to contribute to antipsychotic-induced weight gain [85, 86]. Most studies have shown adiponectin levels to be lower in schizophrenic patients with MetS compared with healthy controls or schizophrenic patients without MetS [87-89], although other studies have shown higher levels of adiponectin [84]. However, there may be a significant effect of antipsychotic treatment, as lower levels of adiponectin and weight gain after taking atypical antipsychotics are associated with higher risk of MetS [89].

Several studies have investigated the effects of antipsychotics on inflammation; however, the results have been inconsistent largely because the anti-inflammatory effects of antipsychotics may vary based on whether the drug is a typical or atypical antipsychotic. For instance, typical antipsychotic drugs, such as haloperidol and perazine, decrease proinflammatory cytokines (IL-1 $\beta$, IL-6, and TNF- $\alpha$ ) in patients with schizophrenia [90]. However, atypical antipsychotics, such as clozapine, are associated with enhanced inflammatory responses [91, 92]. Additionally, in a post-hoc analysis of the Clinical Antipsychotic Trials of Intervention Effectiveness (CATIE) study, the atypical antipsychotic olanzapine significantly enhanced C-reactive protein (CRP) levels after 18 months of treatment [93].

\section{Anxiety}

The relationship between anxiety and MetS has not been clearly established. Multiple cross-sectional studies found no relationship between anxiety symptoms and MetS [94-97]. Longitudinal studies have yielded conflicting results. A small longitudinal study of Finnish women showed that the presence of MetS at baseline predicted the development of anxiety symptoms during a follow-up period of 7 years [98]. However, the presence of anxiety symptoms at baseline did not predict the development of MetS during the study period. Results from the Women's Ischemia Syndrome Evaluation Study also did not find an association between anxiety symptoms and MetS [99].

An even smaller number of studies have examined the relationship between anxiety and proinflammatory biomarkers or adipokines. Liukonnen et al. examined whether anxiety symptoms were associated with low-grade inflammation in a Finnish population [100]. After adjusting for confounders, anxiety symptoms increased the probability for elevated CRP levels in males more than 2-fold, whereas comorbid anxiety and depressive symptoms caused a 1.7-fold increase in the probability for elevated CRP levels. A recent analysis from the Netherlands Study of Depression and Anxiety found elevated levels of CRP in men, but not in women, with a current anxiety disorder compared with controls; there was no association of anxiety with IL-6 or TNF- $\alpha$ [101]. Pitsavos et al. found a positive correlation between anxiety symptoms and levels of CRP, IL- 6 and TNF- $\alpha$ in Greek men, and a positive correlation between anxiety symptoms and levels of CRP in women [102]. A U.S. study also found an association of anxiety symptoms with elevated CRP; however, these associations were attenuated after adjusting for demographic and health-related covariates [103]. 
Finally, few studies have examined the association of inflammatory biomarkers or adipokines with anxiety in the setting of obesity or MetS. Capuron et al. examined the relationship between adiposity, inflammation, and emotional status in obese women awaiting gastric surgery. The authors found that IL- 6 and CRP levels were strongly associated with anxiety symptoms in obese participants, even after controlling for BMI; however, leptin levels were not associated with anxiety symptoms in this cohort [104]. Higher levels of phobic anxiety were associated with higher leptin and TNF- $\alpha$ levels in 984 women with type 2 diabetes from the Nurses' Health Study; however, there was no association with adiponectin [105]. Further research is needed to examine the relationship of inflammation with anxiety symptoms in subjects with MetS.

\section{Conclusions}

Considerable data suggest that individuals with neuropsychiatric disorders tend to have excess CVD morbidity and mortality, largely due to high rates of smoking, central obesity, and diabetes mellitus. Furthermore, the use of certain psychotropic drugs worsens these metabolic disorders, further exacerbating obesity, dyslipidemia, glucose intolerance, and the MetS. To date, it remains unclear whether the chronic inflammation associated with the underlying metabolic abnormalities predisposes to mental illness, or whether chronic inflammation associated with mental illness leads to lifestyles that that then predispose to excess CVD morbidity. Regardless, persons with certain psychiatric illnesses are at such high risk for CVD that screening for traditional risk factors, such as waist circumference, blood pressure, and plasma glucose, is currently recommended. Although additional screening using proinflammatory biomarkers, cytokines, and adipokines is not currently standard of care, there is growing evidence that these molecules provide important information regarding severity of both metabolic as well as psychiatric illness, particularly in obese individuals. It is not unreasonable to think that future screening recommendations might incorporate inflammatory biomarkers for complete assessment of a patient's metabolic and psychiatric risk.

\section{Compliance with Ethics Guidelines}

Conflict of Interest Alanna A. Morris declares that she has no conflict of interest.

Viola Vaccarino declares that she has no conflict of interest.

Human and Animal Rights and Informed Consent This article does not contain any studies with human or animal subjects performed by any of the authors.

\section{References}

Papers of particular interest, published recently, have been highlighted as:

- Of importance

•. Of major importance

1. Ogden CL, Carroll MD, Kit BK, Flegal KM. Prevalence of Obesity in the United States, 2009-2010. NCHS Data Brief No. 82;2013.

2. Ervin RB. Prevalence of metabolic syndrome among adults 20 years of Age and over, by sex, age, race and ethnicity, and body mass index: United States, 2003-2006. Natl Health Stat Rep. 2009;13:1-7.

3. Executive summary of the third report of the national cholesterol education program (NCEP) expert panel on detection, evaluation, and treatment of high blood cholesterol in adults (Adult Treatment Panel III). JAMA 2001;285:2486-97.

4. Grundy SM, Brewer HB, Cleeman JI, Smith SC, Lenfant C, Participants ftC. Definition of Metabolic Syndrome: Report of the National Heart, Lung, and Blood Institute/American Heart Association Conference on Scientific Issues Related to Definition. Circulation. 2004;109:433-8.

5. • Mottillo S, Filion KB, Genest J, et al. The Metabolic Syndrome and Cardiovascular Risk: A Systematic Review and Meta-Analysis. J Am Coll Cardiol. 2010;56:1113-32. This article presents a current analysis of MetS with $C V$ risk and all-cause mortality in $>951,083$ patients.

6. Gami AS, Witt BJ, Howard DE, et al. Metabolic Syndrome and Risk of Incident Cardiovascular Events and Death: A Systematic Review and Meta-Analysis of Longitudinal Studies. J Am Coll Cardiol. 2007;49:403-14.

7. Després J-P, Lemieux I. Abdominal obesity and metabolic syndrome. Nature. 2006;444:881-7.

8. Bays HE, Gonzalez-Campoy JM, Bray GA, et al. Pathogenic potential of adipose tissue and metabolic consequences of adipocyte hypertrophy and increased visceral adiposity. Expert Rev Cardiovasc Ther. 2008;6:343-68.

9. Weisberg SP, McCann D, Desai M, Rosenbaum M, Leibel RL, Ferrante AW. Obesity is associated with macrophage accumulation in adipose tissue. J Clin Invest. 2003;112:1796-808.

10. Despres J-P. Is visceral obesity the cause of the metabolic syndrome? Ann Med. 2006;38:52-63.

11. - Neeland IJ, Ayers CR, Rohatgi AK, et al. Associations of visceral and abdominal subcutaneous adipose tissue with markers of cardiac and metabolic risk in obese adults. Obesity 2013;21(9): 439-47. This article presents important differences in the inflammatory and adverse metabolic profiles of visceral versus subcutaneous adipose.

12. Sattar N, Gaw A, Scherbakova O, et al. Metabolic syndrome with and without $\mathrm{C}$-reactive protein as a predictor of coronary heart disease and diabetes in the west of Scotland Coronary Prevention Study. Circulation. 2003;108:414-9.

13. Ridker PM, Buring JE, Cook NR, Rifai N. C-reactive protein, the metabolic syndrome, and risk of incident cardiovascular events: an 8-year follow-up of 14719 initially healthy American women. Circulation. 2003;107:391-7.

14. Tuomisto K, Jousilahti P, Sundvall J, Pajunen P, Salomaa V. Creactive protein, interleukin- 6 and tumor necrosis factor alpha as predictors of incident coronary and cardiovascular events and total mortality. A population-based, prospective study. Thromb Haemost. 2006;95:511-8.

15. Volpato S, Guralnik JM, Ferrucci L, et al. Cardiovascular disease, interleukin-6, and risk of mortality in older women: the Women's Health and Aging Study. Circulation. 2001;103:947-53. 
16. - Schnabel RB, Yin X, Larson MG, et al. Multiple inflammatory biomarkers in relation to cardiovascular events and mortality in the community. Arterioscler Thromb Vasc Biol. 2013;33:1728-33. This article examined 11 established and novel biomarkers of inflammation and oxidative stress in relation to incident major CVD and mortality in the Framingham Heart Study.

17. Van Guilder GP, Hoetzer GL, Greiner JJ, Stauffer BL, DeSouza CA. Influence of metabolic syndrome on biomarkers of oxidative stress and inflammation in obese adults. Obesity. 2006;14:2127-31.

18. Langenberg C, Bergstrom J, Scheidt-Nave C, Pfeilschifter J, Barrett-Connor E. Cardiovascular death and the metabolic syndrome: role of adiposity-signaling hormones and inflammatory markers. Diabetes Care. 2006;29:1363-9.

19. Greenberg AS, Obin MS. Obesity and the role of adipose tissue in inflammation and metabolism. Am J Clin Nutr. 2006;83:461S-5S.

20. Berg AH, Scherer PE. Adipose tissue, inflammation, and cardiovascular disease. Circ Res. 2005;96:939-49.

21. Maury E, Brichard SM. Adipokine dysregulation, adipose tissue inflammation and metabolic syndrome. Mol Cell Endocrinol. 2010;314:1-16.

22. • Morris AA, Ahmed Y, Stoyanova N, et al. The association between depression and leptin is mediated by adiposity. Psychosom Med. 2012;74:483-8. This article found that the relationship between leptin and depression appears to be mediated by adipostiy, and showed a clinical role for both leptin resistance as well as insufficiency in depressive symptoms.

23. Brennan AM, Mantzoros CS. Drug insight: the role of leptin in human physiology and pathophysiology - emerging clinical applications. Nat Rev Endocrinol. 2006;2:318-27.

24. Loffreda S, Yang SQ, Lin HZ, et al. Leptin regulates proinflammatory immune responses. FASEB J. 1998;12:57-65.

25. Arita Y, Kihara S, Ouchi N, et al. Adipocyte-derived plasma protein adiponectin acts as a platelet-derived growth factor-BB-binding protein and regulates growth factor-induced common postreceptor signal in vascular smooth muscle cell. Circulation. 2002;105:2893-8.

26. Ouedraogo R, Gong Y, Berzins B, et al. Adiponectin deficiency increases leukocyte-endothelium interactions via upregulation of endothelial cell adhesion molecules in vivo. J Clin Invest. 2007;117:1718-26.

27. •- Yirmiya R, Goshen I. Immune modulation of learning, memory, neural plasticity and neurogenesis. Brain Behav Immun. 2011;25: 181-213. This article provides a cellular/molecular model of the role of immune factors in behavioral and neural plasticity in physiological and inflammatory conditions.

28. Raison CL, Capuron L, Miller AH. Cytokines sing the blues: inflammation and the pathogenesis of depression. Trends Immunol. 2006;27:24-31.

29. Hirschfeld R. Depression epidemiology and its treatment evolution. J Clin Psychiatry 2012;73(10):e29.

30. The World Health Organization. The global burden of disease: 2004 update, Table A2: burden of disease in DALYs by cause, sex and income group in WHO regions, estimates for 2004. Geneva, Switzerland: WHO; 2008.

31. - Daly M. The relationship of C-reactive protein to obesity-related depressive symptoms: a longitudinal study. Obesity. 2013;21:24850. This article explores longitudinal associations between obesity, inflammation, and depressive symptoms.

32. • Luppino FS, de Wit LM, Bouvy PF, et al. Overweight, obesity, and depression: a systematic review and meta-analysis of longitudinal studies. Arch Gen Psychiatry. 2010;67:220-9. This article examines the longitudinal bidirectional associations between obesity and depressive symptoms in $>58,000$ patients.

33. Capuron L, Su S, Miller AH, et al. Depressive symptoms and metabolic syndrome: is inflammation the underlying link? Biol Psychiatry. 2008;64:896-900.
34. Kinder LS, Carnethon MR, Palaniappan LP, King AC, Fortmann SP. Depression and the metabolic syndrome in young adults: findings from the third national health and nutrition examination survey. Psychosom Med. 2004;66:316-22.

35. Dunbar JA, Reddy P, Davis-Lameloise N, et al. Depression: an important comorbidity with metabolic syndrome in a general population. Diabetes Care. 2008;31:2368-73.

36. - Lamers F, Vogelzangs N, Merikangas KR, de Jonge P, Beekman ATF, Penninx BWJH. Evidence for a differential role of HPA-axis function, inflammation and metabolic syndrome in melancholic versus atypical depression. Mol Psychiatry. 2013;18:692-9. This article explores important differences in inflammatory markers, diurnal cortisol variations, and MetS components in patients with melancholic versus atypical depression.

37. Vaccarino V, McClure C, Johnson BD, et al. Depression, the metabolic syndrome and cardiovascular risk. Psychosom Med. 2008;70:40-8.

38. Pan A, Keum N, Okereke OI, et al. Bidirectional association between depression and metabolic syndrome: a systematic review and meta-analysis of epidemiological studies. Diabetes Care. 2012;35: $1171-80$.

39. Felger JC, Lotrich FE. Inflammatory cytokines in depression: Neurobiological mechanisms and therapeutic implications. Neuroscience 2013;246:199-229.

40. Lotrich FE. Gene-environment interactions in geriatric depression. Psychiatr Clin N Am. 2011;34:357-76.

41. Charney DS. Monamine dysfunction and the pathophysiology and treatment of depression. J Clin Psychiatry. 1998;59:11-4.

42. Pariante CM, Lightman SL. The HPA axis in major depression: classical theories and new developments. Trends Neurosci. 2008;31:464-8

43. Pariante CM, Miller AH. Glucocorticoid receptors in major depression: relevance to pathophysiology and treatment. Biol Psychiatry. 2001;49:391-404.

44. Miller AH, Maletic V, Raison CL. Inflammation and its discontents: the role of cytokines in the pathophysiology of major depression. Biol Psychiatry. 2009;65:732-41.

45. • Hickman R, Khambaty T, Stewart J. C-reactive protein is elevated in atypical but not nonatypical depression: data from the National Health and Nutrition Examination Survey (NHANES) 1999-2004. J Behav Med 2013; epub 30 Apr 2013:1-9. This article examines CRP levels in adults from the NHANES cohort with atypical, nonatypical and no depression.

46. Elovainio M, Keltikangas J, Auml, et al. Depressive symptoms and C-reactive protein: The Cardiovascular Risk in Young Finns Study. Psychol Med. 2006;36:797-805.

47. Azar R, Nolan R, Stewart D. Listening to the heart-brain talk: persistent depressive symptoms are associated with hsCRP in apparently healthy individuals at high risk for coronary artery disease. Eur J Prev Cardiol. 2012;19:857-63.

48. Danner M, Kasl SV, Abramson JL, Vaccarino V. Association between depression and elevated C-reactive protein. Psychosom Med. 2003;65:347-56.

49. Gimeno D, Kivimaki M, Brunner EJ, et al. Associations of Creactive protein and interleukin- 6 with cognitive symptoms of depression: 12-year follow-up of the Whitehall II study. Psychol Med. 2009;39:413-23. doi:10.1017/S0033291708003723.

50. Elovainio M, Aalto A-M, Kivimaki M, et al. Depression and Creactive protein: population-based health 2000 study. Psychosom Med. 2009;71:423-30.

51. - Janicki Deverts D, Cohen S, DiLillo VG, et al. Depressive symptoms, race, and circulating C-reactive protein: the Coronary Artery Risk Development in Young Adults (CARDIA) study. Psychosom Med. 2010;72:734-41. This article explores racebased differences in the longitudinal association between CRP and depressive symptoms. 
52. - Morris AA, Zhao L, Ahmed Y, et al. Association Between Depression and Inflammation, Differences by Race and Sex: The META-Health Study. Psychosom Med. 2011;73:462-8. This article explores race- and gender-based differences in the association between CRP and depressive symptoms.

53. Penninx BW, Milaneschi Y, Lamers F, Vogelzangs N. Understanding the somatic consequences of depression: biological mechanisms and the role of depression symptom profile. BMC Med. 2013;11:1-14.

54. Su S, Miller AH, Snieder H, et al. Common genetic contributions to depressive symptoms and inflammatory markers in middle-aged Men: the Twins Heart Study. Psychosom Med. 2009;71:152-8.

55. Sonikian M, Metaxaki P, Papavasileiou D, et al. Effects of interleukin-6 on depression risk in dialysis patients. Am J Nephrol. 2010;31:303-8.

56. Bremmer M, Beekman A, Deeg D, et al. Inflammatory markers in late-life depression: results from a population-based study. J Affect Disord. 2008;106:249-55.

57. Pace TWW, Mletzko TC, Alagbe O, et al. Increased stress-induced inflammatory responses in male patients with major depression and increased early life stress. Am J Psychiatry. 2006;163:1630-3.

58. Howren MB, Lamkin DM, Suls J. Associations of depression with C-reactive protein, IL-1, and IL-6: a meta-analysis. Psychosom Med. 2009;71:171-86.

59. • Duivis HE, Vogelzangs N, Kupper N, de Jonge P, Penninx BWJH. Differential association of somatic and cognitive symptoms of depression and anxiety with inflammation: Findings from the Netherlands Study of Depression and Anxiety (NESDA). Psychoneuroendocrinology 2013;38(9):1573-85. This article explores the associatin of both cognitive and somatic symptoms of depression and anxiety with $C R P, I L-6$ and $T N F-\alpha$.

60. Einvik G, Vistnes M, Hrubos-Strom H, et al. Circulating cytokine concentrations are not associated with major depressive disorder in a community-based cohort. Gen Hosp Psychiatry. 2012;34:262-7.

61. - Dowlati Y, Herrmann N, Swardfager W, et al. A meta-analysis of cytokines in major depression. Biol Psychiatry. 2010;67:446-57. This meta-analysis examines the association between depression in 24 studies and multiple cytokines, including $I L-6, T N F-\alpha, I L-1 \beta$, $I L-4, I L-2, I L-8, I L-10$, and $I F N-\gamma$.

62. •- Raison CL, Rutherford RE, Woolwine BJ, et al. A randomized controlled trial of the tumor necrosis factor antagonist Infliximab for treatment-resistant depression: the role of baseline inflammatory biomarkers. JAMA Psychiatry. 2013;70:31-41. This trial examines whether baseline inflammatory biomarkers predict response to use of the TNF- $\alpha$ antagonist infliximab for treatment-resistant depression.

63. Zeugmann S, Quante A, Heuser I, Schwarzer R, Anghelescu I. Inflammatory biomarkers in 70 depressed inpatients with and without the metabolic syndrome. J Clin Psychiatry. 2010;71:1007-16.

64. Jow GM, Yang TT, Chen CL. Leptin and cholesterol levels are low in major depressive disorder, but high in schizophrenia. J Affect Disord. 2006;90:21-7.

65. Kraus T, Haack M, Schuld A, Hinze-Selch D, Pollmacher T. Low leptin levels but Norma body mass indices in patients with depression or schizophrenia. Neuroendocrinology. 2001;73:243-7.

66. Antonijevic IA, Murck H, Frieboes RM, Horn R, Brabant G, Steiger A. Elevated nocturnal profiles of serum leptin in patients with depression. J Psychiatr Res. 1998;32:403-10.

67. Gecici O, Kuloglu M, Atmaca M, et al. High serum leptin levels in depressive disorders with atypical features. Psychiatry Clin Neurosci. 2005;59:736-8.

68. - Chirinos D, Goldberg R, Gellman M, et al. Leptin and its Association with Somatic Depressive Symptoms in Patients with the Metabolic Syndrome. Ann Behav Med 2013;46(1):31-9. This study explored the associatin of leptin with somatic and cognitive symptoms of depression.
69. Lehto SM, Huotari A, Niskanen L, et al. Serum adiponectin and resistin levels in major depressive disorder. Acta Psychiatr Scand. 2010;121:209-15.

70. Leo R, Di Lorenzo G, Tesauro M, et al. Decreased plasma adiponectin concentration in major depression. Neurosci Lett. 2006;407:211-3.

71. - Cizza G, Nguyen VT, Eskandari F, et al. Low 24-hour adiponectin and high nocturnal leptin concentrations in a case control study of community-dwelling premenopausal women with major depression: The P.O.W.E.R. Study. J Clin Psychiatry. 2010;71:1079-87. This article examines the association of adipokines and HPA-axis activation with depression severity.

72. - Hood KK, Lawrence JM, Anderson A, et al. Metabolic and inflammatory links to depression in youth with diabetes. Diabetes Care. 2012;35:2443-6. This article examines eight metabolic and inflammatory markers in youth with type 1 and type 2 diabetes.

73. De Hert M, Schreurs V, Vancampfort D, Van Winkel R. Metabolic syndrome in people with schizophrenia: a review. World Psychiatry. 2009;8:15-22.

74. De Hert M, Van Winkel R, Van Eyck D, et al. Prevalence of diabetes, metabolic syndrome and metabolic abnormalities in schizophrenia over the course of the illness: a cross-sectional study. Clin Pract Epidemiol Ment Health. 2006;2:1-10.

75. De Hert M, Schreurs V, Sweers K, et al. Typical and atypical antipsychotics differentially affect long-term incidence rates of the metabolic syndrome in first-episode patients with schizophrenia: a retrospective chart review. Schizophr Res. 2008;101:295-303.

76. Consensus Development Conference on Antipsychotic Drugs and Obesity and Diabetes. Diabetes Care 2004;27:596-601.

77. Na K-S, Jung H-Y, Kim Y-K. The role of pro-inflammatory cytokines in the neuroinflammation and neurogenesis of schizophrenia. Progress in Neuro-Psychopharmacology and Biological Psychiatry 2012; epub 1 November 2012. doi:10.1016/j.pnpbp.2012.10.022.

78. Miller BJ, Culpepper N, Rapaport MH. C-Reactive Protein Levels in Schizophrenia. Clinical Schizophrenia and Related Disorders 2013; epub 21 February 2013. doi:10.3371/CSRP.MICU.020813.

79. Dickerson F, Stallings C, Origoni A, et al. C-reactive protein is elevated in schizophrenia. Schizophr Res. 2013;143:198-202.

80. Sasayama D, Wakabayashi C, Hori H, et al. Association of plasma IL-6 and soluble IL-6 receptor levels with the Asp358Ala polymorphism of the IL-6 receptor gene in schizophrenic patients. J Psychiatr Res. 2011;45:1439-44.

81. Sasayama D, Hattori K, Wakabayashi C, et al. Increased cerebrospinal fluid interleukin-6 levels in patients with schizophrenia and those with major depressive disorder. J Psychiatr Res. 2013;47:401-6.

82. Zakharyan R, Petrek M, Arakelyan A, Mrazek F, Atshemyan S, Boyajyan A. Interleukin-6 promoter polymorphism and plasma levels in patients with schizophrenia. Tissue Antigens. 2012;80: 136-42.

83. Miller BJ, Buckley P, Seabolt W, Mellor A, Kirkpatrick B. Metaanalysis of cytokine alterations in schizophrenia: clinical status and antipsychotic effects. Biol Psychiatry. 2011;70:663-71.

84. Beumer W, Drexhage RC, De Wit H, Versnel MA, Drexhage HA, Cohen D. Increased level of serum cytokines, chemokines and adipokines in patients with schizophrenia is associated with disease and metabolic syndrome. Psychoneuroendocrinology. 2012;37: 1901-11.

85. Gregoor JG, van der Weide J, Loovers HM, van Megen HJGM, Egberts TCG, Heerdink ER. Polymorphisms of the LEP, LEPR and HTR2C gene: obesity and BMI change in patients using antipsychotic medication in a naturalistic setting. Pharmacogenomics. 2011;12:919-23.

86. Gregoor JG, van der Weide J, Mulder H, et al. Polymorphisms of the LEP- and LEPR gene and obesity in patients using antipsychotic medication. J Clin Psychopharmacol. 2009;29:21-5. 
87. Hanssens L, van Winkel R, Wampers M, et al. A cross-sectional evaluation of adiponectin plasma levels in patients with schizophrenia and schizoaffective disorder. Schizophr Res. 2008;106:308-14.

88. Chen P-Y, Huang M-C, Chiu C-C, Liu H-C, Lu M-L, Chen C-H. Association of plasma retinol-binding protein-4, adiponectin, and high molecular weight adiponectin with metabolic adversities in patients with schizophrenia. Prog Neuro-Psychopharmacol Biol Psychiatry. 2011;35:1927-32.

89. Bai YM, Chen TT, Yang W-S, et al. Association of adiponectin and metabolic syndrome among patients taking atypical antipsychotics for schizophrenia: A cohort study. Schizophr Res. 2009;111:1-8.

90. Kowalski J, Blada P, Kucia K, Madej A, Herman ZS. Neuroleptics normalize increased release of interleukin- 1 beta and tumor necrosis factor-alpha from monocytes in schizophrenia. Schizophr Res. 2001;50:169-75.

91. Contreras-Shannon V, Heart DL, Paredes RM, et al. ClozapineInduced Mitochondria Alterations and Inflammation in Brain and Insulin-Responsive Cells. PLoS One. 2013;8:1-10.

92. Maes M, Bosmans E, Kenis G, De Jong R, Smith RS, Meltzer HY. In vivo immunomodulatory effects of clozapine in schizophrenia. Schizophr Res. 1997;26:221-5.

93. Meyer JM, McEvoy JP, Davis VG, et al. Inflammatory markers in schizophrenia: comparing antipsychotic effects in phase 1 of the clinical antipsychotic trials of intervention effectiveness study. Biol Psychiatry. 2009;66:1013-22.

94. Skilton MR, Moulin P, Terra J-L, Bonnet F. Associations between anxiety, depression, and the metabolic syndrome. Biol Psychiatry. 2007;62:1251-7.

95. Herva A, Rasanen P, Miettunen J, et al. Co-occurrence of metabolic syndrome with depression and anxiety in young adults: the northern Finland 1966 birth cohort study. Psychosom Med. 2006;68:213-6.

96. Takeuchi T, Nakao M, Nomura K, Yano E. Association of metabolic syndrome with depression and anxiety in Japanese men. Diabetes Metab. 2009;35:32-6.
97. de Winter CF, Hermans H, Evenhuis HM, Echteld MA. Associations of symptoms of anxiety and depression with diabetes and cardiovascular risk factors in older people with intellectual disability. Journal of Intellectual Disability Research 2013; epub 30 Apr 2013. doi:10.1111/jir.12049.

98. Raikkonen K, Matthews KA, Kuller LH. The relationship between psychological risk attributes and the metabolic syndrome in healthy women: antecedent or consequence? Metabolism. 2002;51:1573-7.

99. Whittaker KS, Krantz DS, Rutledge T, et al. Combining psychosocial data to improve prediction of cardiovascular disease risk factors and events: the national heart, lung, and blood institute sponsored Women's ischemia syndrome evaluation study. Psychosom Med. 2012;74:263-70.

100. Liukkonen T, Rasanen P, Jokelainen J, et al. The association between anxiety and C-reactive protein (CRP) levels: results from the Northern Finland 1966 Birth Cohort Study. Eur Psychiatry. 2011;26:363-9.

101. Vogelzangs N, Beekman ATF, de Jonge P, Penninx BWJH. Anxiety disorders and inflammation in a large adult cohort. Translational Psychiatry 2013;3:e249.

102. Pitsavos C, Panagiotakos D, Papageorgiou C, Tsetsekou E, Soldatos $\mathrm{C}$, Stefanadis C. Anxiety in relation to inflammation and coagulation markers, among healthy adults : the ATTICA study. Atherosclerosis. 2006;185:320-6.

103. Copeland WE, Shanahan L, Worthman C, Angold A, Costello EJ. Generalized anxiety and C-reactive protein levels: a prospective, longitudinal analysis. Psychol Med. 2012;42:2641-50.

104. Capuron L, Poitou C, Machaux-Tholliez D, et al. Relationship between adiposity, emotional status and eating behaviour in obese women: role of inflammation. Psychol Med. 2011;41: 1517-28.

105. Brennan AM, Fargnoli JL, Williams CJ, et al. s. Diabetes Care. 2009;32:926-31. 